Algebra and Discrete Mathematics Vol. 2

\title{
GRASSMANNIANS OF CLASSICAL BUILDINGS
}

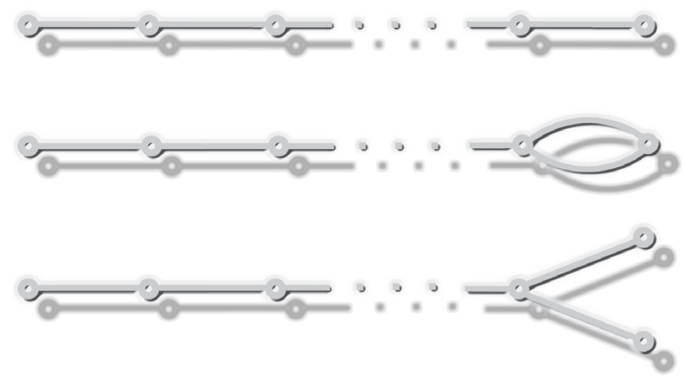




\section{Algebra and Discrete Mathematics}

Managing Editor: Rüdiger Göbel (University Duisburg-Essen, Germany)

Editorial Board: Elisabeth Bouscaren, Manfred Droste, Katsuya Eda, Emmanuel Dror Farjoun, Angus MacIntyre, H.Dugald Macpherson, José Antonio de la Peña, Luigi Salce, Mark Sapir, Lutz Strüngmann, Simon Thomas

The series ADM focuses on recent developments in all branches of algebra and topics closely connected. In particular, it emphasizes combinatorics, set theoretical methods, model theory and interplay between various fields, and their influence on algebra and more general discrete structures. The publications of this series are of special interest to researchers, post-doctorals and graduate students. It is the intention of the editors to support fascinating new activities of research and to spread the new developments to the entire mathematical community.

Vol. 1: Aspects of Infinite Groups: A Festschrift in Honor of Anthony Gaglione eds. Benjamin Fine, Gerhard Rosenberger \& Dennis Spellman

Vol. 2: Grassmannians of Classical Buildings by Mark Pankov 


\title{
GRASSMANNIANS OF CLASSICAL BUILDINGS
}

\author{
Mark Pankov
of Warmia and Mazury, Poland \\ Mark Pankov
University of Warmia and Mazury, Poland
}


Published by

World Scientific Publishing Co. Pte. Ltd.

5 Toh Tuck Link, Singapore 596224

USA office: 27 Warren Street, Suite 401-402, Hackensack, NJ 07601

UK office: 57 Shelton Street, Covent Garden, London WC2H 9HE

\section{British Library Cataloguing-in-Publication Data}

A catalogue record for this book is available from the British Library.

\section{GRASSMANNIANS OF CLASSICAL BUILDINGS Algebra and Discrete Mathematics - Vol. 2}

Copyright (C) 2010 by World Scientific Publishing Co. Pte. Ltd.

All rights reserved. This book, or parts thereof, may not be reproduced in any form or by any means, electronic or mechanical, including photocopying, recording or any information storage and retrieval system now known or to be invented, without written permission from the Publisher.

For photocopying of material in this volume, please pay a copying fee through the Copyright Clearance Center, Inc., 222 Rosewood Drive, Danvers, MA 01923, USA. In this case permission to photocopy is not required from the publisher.

ISBN-13 978-981-4317-56-6

ISBN-10 981-4317-56-X

Printed in Singapore. 


\section{Dedicated}

to

my wife Inna

and

daughters Sabina and Varvara 
This page intentionally left blank 


\section{Preface}

Tits buildings or simple buildings are combinatorial constructions successfully exploited to study various types of groups (classical, simple algebraic, Kac-Moody). One of historical backgrounds of this concept is Cartan's well-known classification of simple Lie groups. We refer to [Abramenko (1996); Brown (1989); Garrett (1997); Ronan (1989); Scharlau (1995); Tits (1974)] for various aspects of building theory.

Buildings can be obtained from groups admitting Tits systems. Such groups form a sufficiently wide class which contains classical groups, reductive algebraic groups and others. The formal definition of a building is pure combinatorial and does not depend on a group. In [Tits (1974)] a building is defined as a simplicial complex with a family of subcomplexes called apartments and satisfying certain axioms. All apartments are isomorphic to the simplicial complex obtained from a Coxeter system which defines the building type.

The vertex set of a building can be labeled by the nodes of the diagram of the associated Coxeter system. The set of all vertices corresponding to the same node is called a Grassmannian (more general objects were investigated in [Pasini (1994)]). This term is motivated by the fact that every building of type $\mathrm{A}_{n}$ is isomorphic to the flag complex of an $(n+1)$ dimensional vector space and the Grassmannians of the building can be identified with the Grassmannians of this vector space. Every building Grassmannian has a natural structure of a partial linear space (point-line geometry); this partial linear space is called the Grassmann space associated with the Grassmannian.

The aim of this book is to present both classical and more recent results on Grassmannians of buildings of classical types $\left(\mathrm{A}_{n}, \mathrm{~B}_{n}=\mathrm{C}_{n}, \mathrm{D}_{n}\right)$. These results will be formulated in terms of point-line geometry. A large 
portion of them is a part of the area known as characterizations of geometrical transformations under mild hypotheses. Roughly speaking, we want to show that some mappings of Grassmannians can be extended to mappings of the associated buildings. Other results are related with structural properties of apartments. Also we show that our methods work for some geometric constructions non-related with buildings - Grassmannians of infinity-dimensional vector spaces, the sets of conjugate linear involutions and Grassmannians of exchange spaces.

The book is self-contained and prospective audience includes researchers working in algebra, combinatorics and geometry, as well as, graduate and advanced undergraduate students. The requirement to the reader is knowledge of basics of algebra and graph theory.

\section{Acknowledge}

The present book contains the main results of my Doctor of Science (habilitation) thesis presented in Institute of Mathematics NASU and I thank A. Samoilenko and V. Sharko for supporting my research.

I am grateful to H. Van Maldeghem who read a preliminary version of the book and made a long list of valuable comments. Also I am grateful to H. Havlicek and M. Kwiatkowski for useful discussions and remarks.

Finally, I thank J. Kosiorek for drawing the pictures. 


\section{Contents}

Preface vii

0. Introduction 1

$\begin{array}{ll}\text { 1. Linear Algebra and Projective Geometry } & 7\end{array}$

1.1 Vector spaces . . . . . . . . . . . . . . 8

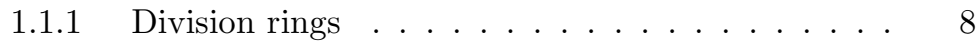

1.1.2 Vector spaces over division rings . . . . . . . . . . 10

1.1 .3 Dual vector space . . . . . . . . . . . . . . . . . . 14

$1.2 \quad$ Projective spaces . . . . . . . . . . . . . . . 17

1.2.1 Linear and partial linear spaces . . . . . . . . 17

1.2.2 Projective spaces over division rings . . . . . . . . 19

1.3 Semilinear mappings . . . . . . . . . . . . . . . . 20

1.3.1 Definitions . . . . . . . . . . . . 20

1.3.2 Mappings of Grassmannians induced by semilinear

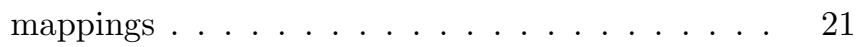

1.3.3 Contragradient. . . . . . . . . . . . 25

1.4 Fundamental Theorem of Projective Geometry . . . . . . 26

1.4.1 Main theorem and corollaries . . . . . . . . . 26

1.4.2 Proof of Theorem 1.4 . . . . . . . . . . 28

1.4.3 Fundamental Theorem for normed spaces . . . . . 32

1.4.4 Proof of Theorem 1.5 . . . . . . . . . . . . 33

1.5 Reflexive forms and polarities . . . . . . . . . . . . 37

1.5.1 Sesquilinear forms . . . . . . . . . . . . . . . . . . 37

1.5.2 Reflexive forms . . . . . . . . . . . . 38

1.5.3 Polarities . . . . . . . . . . . . . . 40 
2. Buildings and Grassmannians 43

2.1 Simplicial complexes . . . . . . . . . . . . . . . . 43

2.1.1 Definition and examples . . . . . . . . . . 43

2.1.2 Chamber complexes . . . . . . . . . . . . 46

2.1.3 Grassmannians and Grassmann spaces . . . . . . 47

2.2 Coxeter systems and Coxeter complexes . . . . . . . . . 49

2.2 .1 Coxeter systems . . . . . . . . . . . . . . 49

2.2 .2 Coxeter complexes . . . . . . . . . . . . . 52

2.2 .3 Three examples . . . . . . . . . . . 53

2.3 Buildings . . . . . . . . . . . . . . 55

2.3.1 Definition and elementary properties . . . . . 55

2.3.2 Buildings and Tits systems . . . . . . . . . . 57

2.3.3 Classical examples . . . . . . . . . . . . . . . 59

2.3.4 Spherical buildings . . . . . . . . . . . 62

2.3.5 Mappings of the chamber sets . . . . . . . . 63

2.4 Mappings of Grassmannians . . . . . . . . . . . . . . . . 65

2.5 Appendix: Gamma spaces . . . . . . . . . . . . 67

3. Classical Grassmannians $\quad 69$

3.1 Elementary properties of Grassmann spaces . . . . . . . 70

3.2 Collineations of Grassmann spaces . . . . . . . . . . 75

3.2.1 Chow's theorem . . . . . . . . . . . . 75

3.2.2 Chow's theorem for linear spaces . . . . . . . . . 77

3.2.3 Applications of Chow's theorem . . . . . . . . 78

3.2 .4 Opposite relation . . . . . . . . . . . . 80

3.3 Apartments .................... 83

3.3.1 Basic properties . . . . . . . . . . 83

3.3.2 Proof of Theorem $3.8 \ldots \ldots \ldots \ldots$

3.4 Apartments preserving mappings . . . . . . . . . 87

3.4 .1 Results . . . . . . . . . . . . . 87

3.4.2 Proof of Theorem 3.10: First step . . . . . . . 89

3.4.3 Proof of Theorem 3.10: Second step . . . . . . . . 93

3.5 Grassmannians of exchange spaces . . . . . . . . . . 95

3.5.1 Exchange spaces . . . . . . . . . . . . . . . 95

3.5.2 Grassmannians . . . . . . . . . . . . . . 96

3.6 Matrix geometry and spine spaces . . . . . . . . 100

3.7 Geometry of linear involutions . . . . . . . . . . . . . . 102

3.7.1 Involutions and transvections . . . . . . . . . . 102 
3.7.2 Adjacency relation . . . . . . . . . . . . . . 104

3.7.3 Chow's theorem for linear involutions . . . . . . . 108

3.7.4 Proof of Theorem 3.15 . . . . . . . . . . . 110

3.7.5 Automorphisms of the group GL $(V) \ldots \ldots \ldots$

3.8 Grassmannians of infinite-dimensional vector spaces . . . 114

3.8.1 Adjacency relation . . . . . . . . . . . . . . 114

3.8.2 Proof of Theorem $3.17 \ldots \ldots \ldots$

3.8.3 Base subsets . . . . . . . . . . . . . . 118

3.8.4 Proof of Theorem $3.18 \ldots \ldots \ldots \ldots$

4. Polar and Half-Spin Grassmannians 123

$4.1 \quad$ Polar spaces . . . . . . . . . . . . . . . . . . 125

4.1.1 Axioms and elementary properties . . . . . . . 125

4.1.2 Proof of Theorem $4.1 \ldots \ldots$. . . . . . . 126

4.1.3 Corollaries of Theorem 4.1 . . . . . . . . . . . . 129

4.1.4 Polar frames . . . . . . . . . . . . . . . . . 130

4.2 Grassmannians . . . . . . . . . . . . . . . . . 133

4.2.1 Polar Grassmannians . . . . . . . . . . . . . 133

4.2.2 Two types of polar spaces _ . . . . . . . . 136

4.2.3 Half-spin Grassmannians . . . . . . . . . . . . . 138

4.3 Examples . . . . . . . . . . . . . . . . . . 141

4.3.1 Polar spaces associated with sesquilinear forms . . 141

4.3.2 Polar spaces associated with quadratic forms . . . 146

4.3.3 Polar spaces of type $\mathrm{D}_{3} \ldots \ldots \ldots \ldots$. . . . . . 147

4.3.4 Embeddings in projective spaces and classification 149

4.4 Polar buildings . . . . . . . . . . . . . . . . . . 150

4.4 .1 Buildings of type $C_{n} \ldots \ldots \ldots \ldots$

4.4.2 Buildings of type $\mathrm{D}_{n} \ldots \ldots \ldots \ldots$

4.5 Elementary properties of Grassmann spaces . . . . . . . . 151

4.5.1 Polar Grassmann spaces . . . . . . . . . . . . 151

4.5.2 Half-spin Grassmann spaces . . . . . . . . . . . 154

4.6 Collineations . . . . . . . . . . . . . . . . . . 159

4.6.1 Chow's theorem and its generalizations . . . . . . 159

4.6.2 Weak adjacency on polar Grassmannians . . . . . 161

4.6.3 Proof of Theorem 4.8 for $k<n-2 \ldots \ldots$. . . . 162

4.6.4 Proof of Theorems 4.7 and $4.8 \ldots \ldots \ldots$. . . . 164

4.6.5 Proof of Theorem $4.9 \ldots \ldots \ldots$

4.6.6 Remarks . . . . . . . . . . . . . . . . . 172

4.7 Opposite relation . . . . . . . . . . . . . . . . . 174 
4.7.1 Opposite relation on polar Grassmannians . . . . 174

4.7.2 Opposite relation on half-spin Grassmannians . 175

4.8 Apartments . . . . . . . . . . . . . . . 178

4.8.1 Apartments in polar Grassmannians . . . . . . . . 178

4.8.2 Apartments in half-spin Grassmannians . . . . . . 181

4.8.3 Proof of Theorem 4.15 . . . . . . . . . . . . . . 184

4.9 Apartments preserving mappings . . . . . . . . . . 186

4.9.1 Apartments preserving bijections . . . . . . . 186

4.9.2 Inexact subsets of polar Grassmannians . . . . . . 187

4.9.3 Complement subsets of polar Grassmannians . . . 194

4.9.4 Inexact subsets of half-spin Grassmannians . . . . 199

4.9.5 Proof of Theorem 4.16 . . . . . . . . . . . . 201

4.9.6 Embeddings . . . . . . . . . . . . . . 202

4.9.7 Proof of Theorems 4.17 and $4.18 \ldots \ldots$. . . . 203

$\begin{array}{ll}\text { Bibliography } & 207\end{array}$

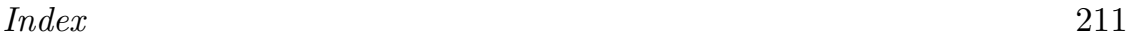

